\title{
An Application of Laplace Transform, Inverse Laplace Transform and Pade's Approximant in the Periodic Solution of Duffing Equation of Motion
}

\author{
J.Peter Praveen ${ }^{1}$, B.Nageswara Rao ${ }^{2}$, B.Mahaboob ${ }^{3}$, B.V.Appa Rao ${ }^{4}$ \\ ${ }^{1}$ Department of Mathematics, Koneru Lakshmaiah Education Foundation, Vaddeswaram, Guntur-522502, \\ *jppraveen17@kluiversity.in \\ ${ }^{2}$ Department of Mechanical Engineering, Koneru Lakshmaiah Education Foundation, Vaddeswaram, Guntur- \\ 522502, bnrao52@rediffmail.com \\ ${ }^{3}$ Department of Mathematics, Koneru Lakshmaiah Education Foundation, Vaddeswaram, Guntur-522502, \\ mahaboob@kluiversity.in \\ ${ }^{4}$ Department of Mathematics, Koneru Lakshmaiah Education Foundation, Vaddeswaram, Guntur-522502, \\ bvardr2010@kluiversity.in
}

\begin{abstract}
The main objective of this research is to obtain the periodic solution by inserting the ideas of Laplace Transformation, Pade's Approximant and Inverse Laplace Transformation are applied. In this research article the Standard Duffing Equation of motion possessing symmetric oscillations is examined by using the Sumudu Transform Series Decomposition Method. The Duffing oscillator is a periodically forced oscillator with a nonlinear elasticity. For this type of oscillatory system there are frequencies at which the vibration suddenly jumps and these jumps depend upon whether the frequency is increasing or decreasing. Between these frequencies, multiple solutions exist for a given frequency of excitation, and the initial conditions determine which of these solutions represents the response of the system. The Sumudu Transform Series Decomposition Method (STSDM) is claimed as an efficient method for solving problems in Engineering and Science fields. It gives divergent series solution.

This article explores on studying the application capacity of STSDM in getting solution for symmetric oscillations of Duffing Equation of Motion which are simple and nonlinear.
\end{abstract}

Key words: Sumudu Transform (ST); Inverse Sumudu Transform (IST), Adomian Polynomials; Duffing Equation, Truly Nonlinear Oscillators, Laplace Transform (LT), Inverse Laplace Transform (ILT); Pade's Approximant (PA).

\section{INTRODUCTION}

A nonlinear second-order ordinary differential equation of the Duffing oscillator is [1-15].

$$
\ddot{x}+\xi x+g(x)=G_{0}(t)
$$

Over dot denote differentiation with respect to time, $t$. $\xi$ is the damping factor. $G_{0}(t)$ is a time dependent forcing function. The cubic polynomial restoring force function is

$$
g(x)=a x+b x^{2}+c x^{3}+d
$$

Eq. (2) represents:

Asymmetric oscillations for $b \neq 0$ and/or $d \neq 0$. Symmetric oscillations for $b=d=0$.

$c>0$, is for the hardening type of the system.

$c<0$, is for the softening type of the system.

STSDM is considered to be an efficient tool for cracking a large number of physical problems in Science and Engineering. Sumudu Transform reduces the complexity in the integration of highly integral functions which are nonlinear. The rate of convergence increases the solution's series expansion. Nonlinear terms in the DE are resolved by the Adomian Polynomials.

This paper studies the application capacity of STSDM in getting solution for symmetric oscillations of Duffing Equation of Motion which are simple and nonlinear.

\section{ANALYSIS}

A Duffing Equation of Motion which is simple and nonlinear is obtained by

$$
\begin{aligned}
& \dot{x}+x+0.3 x^{3}=0 \\
& x=0 \text { and } \dot{x}=1 \text { at } t=0
\end{aligned}
$$

the restoring force function is $g(x)=x+0.3 x^{3}$. If (3) and (4) are applied by ST[18-23], one can see

$S\{x(t)\}=v-v^{2} S\left[x(t)+0.3 x^{3}(t)\right]$

If (5) is applied by IST, the result becomes 
$x(t)=t-S^{-1}\left[v^{2} S\left(x(t)+0.3 x^{3}(t)\right)\right]$

Assuming the series solution $x(t)=\sum_{m=0}^{\infty} x_{m}(t)$ for Eq.(3) and expressing (6) as

$$
\sum_{m=0}^{\infty} x_{m}(t)=t-S^{-1}\left[v^{2} S\left(\sum_{m=0}^{\infty} A_{m}\right)\right]
$$

The Adomian Polynomial Functions in (7) are

$$
\begin{gathered}
A_{m}=\left.\frac{1}{m !} \frac{d^{m}}{d \theta^{m}}\left[\sum_{j=0}^{\infty} \theta^{j} x_{j}+0.3\left(\sum_{j=0}^{\infty} \theta^{j} x_{j}\right)^{3}\right]\right|_{\theta=0} \\
=x_{m}+0.3 \sum_{j=0}^{\infty} x_{j} x_{m-j}
\end{gathered}
$$

Comparing like terms on both sides and using (8) in (7) one can have

$$
\begin{aligned}
& x_{0}(t)=t \\
& x_{m+1}(t)=-S^{-1}\left[v^{2} S\left(A_{m}\right)\right] \quad m \geq 0 \\
& x_{1}(t)=-\frac{t^{3}}{6} \\
& x_{2}(t)=-0.8 \frac{t^{5}}{120} \\
& x_{3}(t)=18.8 \frac{t^{7}}{5040}
\end{aligned}
$$

the series solution of (3) with boundary constraints (4) are got as

$$
\begin{aligned}
& x(t)=\sum_{m=0}^{\infty} x_{m}(t) \\
& x(t)=t-\frac{t^{3}}{6}-0.8 \frac{t^{5}}{120}+18.8 \frac{t^{7}}{5040}+\ldots .
\end{aligned}
$$

The series solution (15) is unable to exhibit the periodicity.

Applying LT, $[4 / 4]$ PA, and the ILT, the solution of the problem is got as

$x(t)=0.928746 \operatorname{SIN}(1.10982 t)-0.0103828 \operatorname{SIN}(2.96113 t)(16)$

The phase diagram for (1) with the boundary constraints (2) arises as

$$
\begin{aligned}
(\dot{x})^{2} & =1-x^{2}-0.15 x^{4} \\
& =\left(1+0.13246 x^{2}\right)\left(1-1.13246 x^{2}\right)
\end{aligned}
$$

For +ve and -ve amplitudes the behavior oscillations are one and the same. The singular point of $\mathrm{DE}(1)$ in the phase diagram (that is $\dot{x} \mathrm{Vs} \mathrm{x}$ curve) got from the zeros of $\mathrm{g}(\mathrm{x})$ is $(0,0)$. The primitive of $\mathrm{g}(\mathrm{x})$ w.r.t is $\mathrm{x}$ is $g^{\prime}(x)=1+0.3 x^{2}$ and $g(0)=1>0$, which implies that the singular point
$(0,0)$ is turned into centre. If $g^{\prime}\left(x^{*}\right)$ is negative then $x^{*}$ is a saddle point.

Eq.(17)gives the phase diagram ( $\dot{x} \mathrm{Vs}$ x) for the DE (1) with boundary constraints (2). $\dot{x} \mathrm{Vs} \mathrm{x}$ plot arises out of Eq.(17) and this graph depicts the boundary which is closed. Consequently the existence of the periodic solution is being obtained. Eq. (17) depicts the equal magnitude of +ve \& -ve amplitudes (that is $\mathrm{x}=+0.9397$ or $-.09397, \dot{x}=0$ ), when $\mathrm{x}=0, \dot{x}=+1$ or $\dot{x}=-1$. From the STSDM solution (16) one can have $\dot{x}$ values as 1.047,1.016 at $\mathrm{x}=0$. The STSDM gives the solution exactly in the neighborhood of the domain in which boundary constraints are described.

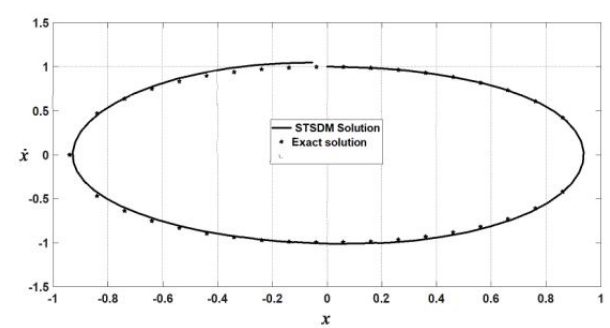

Figure 1 :Phase diagrams of Duffing equation arised out of the solution of STSDM versus Exact Solution

The solution obtained by the STSDM method is divergent in order to make the solution convergent the concept of Laplace transformation and Pade's approximant and Inverse laplace transformation is applied like the procedure followed for the modified differential transform method.

Praveen \& Rao [10], solved the duffing equation of motion having the symmetric oscillations. The solution obtained by the above mentioned procedure gives the solution exactly near the region where the initial conditions are specified. They used the Harmonic balance method to examine the performance of the nonlinear oscillations. The solution obtained by using the Harmonic balance method coincides exact solution.

The following diagram shows the comparison of the solution obtained by Harmonic balance method with the exact solution. 


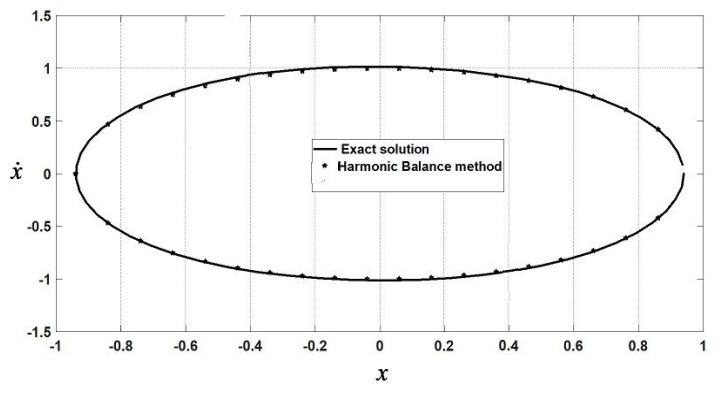

Figure 2 :Phase diagrams of Duffing equation arises out of the solution of Harmonic balance method Vs Exact Solution

The discrepancy in solution (16) is mainly due to the first harmonic frequency and is not three times of third harmonic frequency, which results in different magnitudes. In their study they also suggested some modification to the procedure and obtained the solution and again compared the solution with the exact solution which is able to capture the actual trend in the phase diagram.

The following figure 3 shows the comparison of the solution obtained after modification to the solution obtained by MDTM to the exact solution.

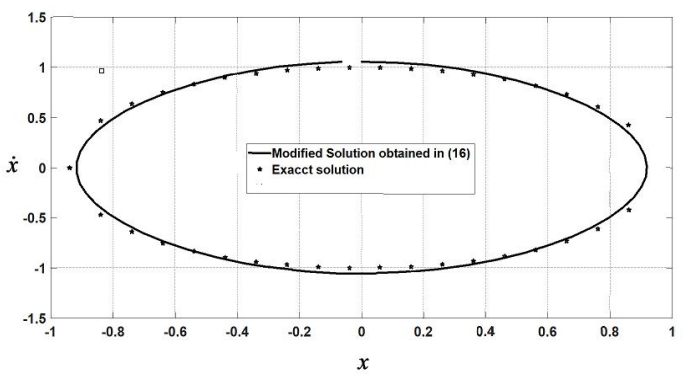

Figure 3: Phase diagrams of Duffing equation arised out of the solution of modified STSDM solution versus Exact Solution

\section{CONCLUSION}

The behaviour of oscillations of the Duffing equation of motion (1) with boundary constraints (2) can easily be understood through generation of phase diagrams. Since the controlling equation is a second order NLODE, one has to verify the approximate solution and its first derivative with the numerical solution. Hence in this research article, the first order differential equation is arrived after integrating the Duffing equation of motion and applying the boundary constarints, which can depicts the exact phase diagram. The periodic solution of the Duffing equation of motion obtained by STSDM is not capturing equal magnitudes of first derivative of $x$ at $x=0, t=0$ and at the period of oscillations. The STSDM provides the solution accurately near to the region where the initial conditions are specified. It follows the actual trend in the phase diagram when the solution (16) is modified.

\section{REFERENCES}

1. M.S. Sarma, A.P. Beena and B. Nageswara Rao, Applicability of perturbation technique to the periodic solution of $x^{3}+\alpha x+\beta x^{2}+\gamma x^{3}=0$, Journal of Sound and Vibration, Vol.180, pp.177$184,1995$.

2. M.S. Sarma, B. Nageswra Rao and H.R. Nataraja, Final solution of Duffing equation of mixedparity, AIAA Journal, Vol.35, No.7, pp.1246$1248,1997$.

3. T. Manoj, M. Ayyappan, K.S. Krishnan and B. Nageswara Rao, Nonlinear vibration analysis of thin laminated rectangular plates on elastic foundations, Z. angew. Math. Mech. (ZAMM), Vol.80, No.3, pp.183-192,2000.

4. R.H. Enns and G.C. Mcgurie, Nonlinear physics with mathematica for scientists and Engineers, Bikhauser, Boston 2001.

5. K. Jayakumar, D. Yadav and B. Nageswara Rao, Nonlinear vibration analysis for a generic coupled-laminated plate with surface bonded or embedded induced strain actuators, Journal of Sound and Vibration, Vol.301, pp.846-863 ,2007.

6. K. Jayakumar, D. Yadav and B. Nageswara Rao, , Non-linear free vibration analysis of generic coupled induced strain actuated piezolaminated beams ,Forschung im Ingenieurwesen, Vol.72,pp.153-162,2008

7. K. Jayakumar, D. Yadav and B. Nageswara Rao, Nonlinear free vibration analysis of a piezolaminated plate with random actuation electric potential difference and material properties, Communications in Nonlinear Science and Numerical Simulations, Vol.14, No.4, pp.1646$1663,2009$.

8. T. Pirbodoghi and G.H. Farrahi, Duffing equations with cubic and quintic nonlinearities, Computers and Mathematics with Applications, Vol. 57, No.3, pp.500-506 ,2009.

9. L. Cveticanin, "Ninety years of Duffing's equation", Theoretical and Applied Mechanics, Belgrade Vol.40, No.1, pp.49-63, 2013.

10. J. Peter Praveen and B. Nageswara Rao, On the periodic solutions of the Helmholtz Equation using the modified differential transform method, MAYFEB Journal of Mathematics, Vol.2, pp.7-16 2016

11. R. Najafi and B.N. Saray, Numerical solution of the forced Duffing equations using Legendre multi-wavelets, Computational Methods for Differential Equations Vol.5, No.1, pp. 43-55, 2017. 
12. J. Sunday, "The Duffing Oscillator: Applications and Computational Simulations", Asian Research Journal of Mathematics Vol.2, No.3, pp.1-13 (2017

13. J. Peter Praveen and B. Nageswara Rao, Uncertainties in the Periodic Solution of a Truly Non-linear Oscillator Differential Equation Using MDTM, International Journal of Applied and Computational Mathematics, Vol.5, No.4, 2019.

14. F.B.M. Belgacem, Introducing and analyzing deeper Sumudu properties, Nonlinear Studies, Vol.13, No.1, pp. 23-41,2006

15. S. Momani and V.S. Erturk, Solutions of nonlinear oscillators by the modified differential transform method, Computers \&Mathematics with Applications, Vol.55, No.4, pp.833-842, 2008.

16. F.B.M.Belgacem, Sumudu transform applications to Bessel functions and equations, Applied Mathematical Sciences, Vol.4, No.74, pp.3665-3686.2010

17. F.B.M. Belgacem and R. Silambarasan Maxwell's equations solutions by means of the natural transform, Mathematics in Engineering, Science and Aerospace (MESA), Vol.3, No.3, pp.313-323,2012.

18. H. Eltayeb, A. K1lic, man, Application of Sumudu Decomposition Method to Solve Nonlinear System of Partial Differential Equations, Abstract and Applied Analysis, Vol. 2012, Article ID 412948, 13 pages 2012.

19. D. Kumar, J. Singh and S. Rathore, Sumudu Decomposition Method for Nonlinear Equations, International Mathematical Forum, Vol. 7, No. 11, pp.515 - 521,2012.

20. F.B.M. Belgacem and R. Silambarasan, Laplace transform analytical restructure, Applied Mathematics, Vol.4, pp.919-932,2013.

21. R. Khandelwal, P. Kumawat and Y. Khandelwal, A study of natural transform based on decomposition method for solving nonlinear ordinary differential equation, International Journal of Statistics and Applied Mathematics, Vol.3, No.2, pp.664-669 2018.

22. Rao T.S., Kumar G.S., Vasavi Ch., Rao B.V.A. (2017), On the controllability of fuzzy difference control systems, International Journal of Civil Engineering and Technology, 8(12), PP.723-732.

23. Ch.Vasavi, G.S.Kumar, T.S.Rao, B.V.A.Rao. (2017), Application of fuzzy differential equations for cooling problems, International Journal of Mechanical Engineering and Technology, 8(12), PP.712-721.

24. P.Bojja, N.M. Prasanna N.M, P.R.Kumari, Development of mathematical model of rotary kiln which are used for design conventional control systems, International Journal of
Engineering and Technology(UAE) ,7(3.12 Special Issue 12), PP. 1182-1186, 2018.

25. P.Kumar, P.S.Keerthika, An inventory model with variable holding cost and partial backlogging under interval uncertainty: Global criteria method, International Journal of Mechanical Engineering and Technology, 9(11), PP. 1567-1578, 2018.

26. T.S.Rao, G.Sureshkumar,M.S.N.Murty M.S.N., $\Psi$ - stability for nonlinear difference equations, Thai Journal of Mathematics ,16(3), PP. 801-815, (2018)

27. J. Peter Praveen, R. Donthi, S.V.Prasad, B. Mahaboob, B.Venkateswarlu B., A glance on the estimation of Cobb-Douglas production functional model, AIP Conference Proceedings, 2177 (1), 020067. 2019

28. J.Peter Praveen, B. Mahaboob, R.Donthi, S.V. Prasad, B.Venkateswarlu, On stochastic linear regression model selection, AIP Conference Proceedings, 2177 (1), 020068, 2019.

29. B.Mahaboob, J.Peter Praveen, R.Donthi, S.V.Prasad, B.Venkateswarlu B., Criteria for selection of stochastic linear model selection, AIP Conference Proceedings, 2177 (1), 020041,2019.

30. R.Donthi, S.V.Prasad, B.Mahaboob, J.Peter Praveen, B.Venkateswarlu. Estimation methods of nonlinear regression models, AIP Conference Proceedings, 2177 (1), 020081, 2019.

31. R.Donthi, J.Peter Praveen, S.V.Prasad, B.Mahaboob, B.Venkateswarlu, Numerical techniques of nonlinear regression model estimation, AIP Conference Proceedings, 2177 (1), 020082. 2019.

32. B.Mahaboob, S.V.Prasad, J.Peter Praveen, R.Donthi, B.Venkateswarlu, On misspecification tests for stochastic linear regression model, AIP Conference Proceedings, 2177 (1), 020039. 2019.

33. B.Mahaboob, Ajmath K.A., Venkateswarlu B., Narayana C., Praveen J.P. ,On Cobb-Douglas production function model, AIP Conference Proceedings, 2177 (1), 020040. 2019.

34. B.Mahaboob, K.A.Azmath, B.Venkateswarlu, C.Narayana, B.M.Rao An evaluation in generalized LSE of linearized stochastic statistical model with non-spherical errors, AIP Conference Proceedings, 2177(1), 020038. 2019.

35. J. Peter Praveen, B. Nageswara Rao, Y. Haranadh, B. V. Appa Rao, C. Narayana,And B. Mahaboob, Existence Of Solution Of The Nonlinear Differential Equation In The Modeling Of Eardrum By Using Homotopy Perturbation Method" Advancecs in mathematics Scientifc journal Vol.9(7), pp.19,2020 
J.Peter Praveen et al., International Journal of Emerging Trends in Engineering Research, 8(9), September 2020, 5979 - 5983

36. A.D. M. Africa, S.A. Pasia, J.A Sy, A Modeling system on the Implementation of Automated Vehicles, International Journal of Emerging Trends in Engineering Research,Vol.8(7),29272933,2020.

37. N.Z. Mahabob, Z.M.Yusoff, N.Ismail, M. N. Taib,. Linear Regression of Gaharu Oil Significant Compounds for Oil Quality Differentiation, International Journal of
Emerging Trends in Engineering Research, Vol.8(7),2893-2900

38. B. Mahaboob, J. Peter Praveen, B. V. Appa Rao, Y. Haranadh, C. Narayana, And G. Balaji Prakash, A Study On Multiple Linear Regression Using Matrix Calculus", Advancecs in mathematics Scientifc journaVol.9(7), pp.1-10 ,2020 